\title{
EFFICIENTLY GENERATED SPACES OF CLASSICAL SIEGEL MODULAR FORMS AND THE BÖCHERER CONJECTURE
}

\author{
MARTIN RAUM \\ (Received 4 July 2010; accepted 11 January 2011) \\ Communicated by F. Calegari
}

\begin{abstract}
We state and verify up to weight 172 a conjecture on the existence of a certain generating set for spaces of classical Siegel modular forms. This conjecture is particularly useful for calculations involving Fourier expansions. Using this generating set, we verify the Böcherer conjecture for nonrational eigenforms and discriminants with class number greater than one. As a further application we verify another conjecture for weights up to 150 and investigate an analog of the Victor-Miller basis. Additionally, we describe some arithmetic properties of the basis we found.
\end{abstract}

2010 Mathematics subject classification: primary 11F46; secondary 11F67, 11 Y99.

Keywords and phrases: Siegel modular forms, Böcherer conjecture, Hecke action, computation.

\section{Introduction}

We know many basic facts about Siegel modular forms of degree two. In particular, the classical Siegel modular forms have been thoroughly investigated. However, we still lack knowledge about analogs of well-known properties of elliptic modular forms. A first effort to overcome this situation using a computational approach was made in [17]. This work focused on scalar-valued (that is, classical) Siegel modular forms. There are recent calculations of Hecke eigenvalues of vector-valued Siegel modular forms by Bergström et al. [1] that use cohomological methods. The effort made to explore paramodular forms of low weight in [13] should also be mentioned. Building on this and the paramodular conjecture (see [5]), some spinor $L$-series attached to paramodular forms were calculated in [16]. This last work is particularly significant since it begins to build the picture for high-level paramodular groups. We also point out that the author will extend the calculations presented in this paper to the paramodular case in another paper.

(C) 2011 Australian Mathematical Publishing Association Inc. 1446-7887/2011 \$16.00 
All the computations mentioned above have in common the feature that they are restricted to low weights. Moreover, they focus on Fourier expansions. But only the last one is able to provide access to the associated $L$-series with sufficient precision. However, this computation relies on the paramodular conjecture and is hence not provably correct-though it is very useful. It is also restricted to weight-2 forms. In contrast, in this paper, we want to consider modular forms of higher weights. We need an efficient way to generate the Fourier expansions of Siegel modular forms for forms of weight $k$ if $k$ is large.

We provide evidence for a conjecture on generating sets for spaces of classical Siegel modular forms. Namely, the products of at most two elements of the Maass Spezialschar form a generating set of the space of all Siegel modular forms associated to the full modular group. We will use Sage [18] for our computations. Additionally, we will use Magma [4] for fast linear algebra.

In Section 2, we verify that these products generate the spaces of Siegel modular forms up to weight 172. This makes it possible to calculate Fourier expansions for very high-weight forms to high precision. These Fourier expansions can be used to compute the attached spinor $L$-series of Hecke eigenforms up to a precision involving primes up to 1500 .

We will do this in Section 3 to investigate the following conjecture. In [2], an analog of the Gross-Zagier theorem for the spinor $L$-series attached to Siegel modular forms of degree two was conjectured.

Conjecture 1 (Böcherer). Let $f(z)=\sum_{t} a_{f}(t) \exp (2 \pi i \cdot \operatorname{tr}(t z))$ be a Siegel Hecke eigenform of degree two associated to the full modular group. Denote by $Z_{f, D}$ the attached spinor $L$-series twisted by the Kronecker character $\left(\frac{D}{.}\right)$, where $D<0$. Then

$$
Z_{f, D}(k-1)=c_{f} B_{f}(D)
$$

where

$$
B_{f}(D)=|D|^{1-k}\left(\sum_{[t] \in S(D)} \frac{a_{f}(t)}{|\operatorname{Aut}(t)|}\right)^{2}
$$

for some $c_{f} \in \mathbb{C}$ depending only on $f$. Here $[t]$ runs over a set $S(D)$ of representatives with respect to the $\mathrm{SL}_{2}(\mathbb{Z})$ action such that $\operatorname{det} t=-D / 4$, and the automorphism group of $t$ is given by

$$
\operatorname{Aut}(t)=\left\{a \in \mathrm{SL}_{2}(\mathbb{Z}): a^{\mathrm{tr}} t a=t\right\} .
$$

A proof of this conjecture could also provide a tool to investigate statistics of central values more efficiently.

The conjecture has been proved for Maass lifts (see [2]) by using the splitting of the $L$-series and Waldspurger's theorem on special values of $L$-series attached to elliptic modular forms. The case of Yoshida lifts of weight two has also been treated (see [3]). The author [14] and Ryan and Tornaría [16] recently argued that the conjecture should also hold in the paramodular setting, and proved that it holds for Gritsenko lifts. For 
forms that are not Maass lifts, it seems inaccessible at the moment. Indeed, even the functional equation of the twisted $L$-series, which appears in the conjecture, has been proved completely only recently [12].

The conjecture has been verified in [10] for even weights $k$ from 20 to 26 and discriminants $D \in\{-3,-4,-7,-8\}$. Besides the small number of cases considered, there are two other outstanding problems with this verification. First, all discriminants correspond to quadratic number fields with class number one. Second, all Hecke eigenforms are rational up to weight 26 . But the space of cuspidal forms that are not Maass lifts has dimension two for weight 24 and 26. Hence the rational Hecke action on this space is not irreducible. This phenomenon is exceptional, as suggested by computations presented in Section 4. More precisely, the rational action of the Hecke algebra on spaces of Siegel modular forms of weight $k$ is irreducible when $28 \leq k \leq 150$. The reason for this curious fact is not yet known, but checking the Böcherer conjecture outside the range of this phenomenon can provide further support for it.

This is done in Section 3. We obtain intervals $I_{f, D}$ such that $c_{f}$ (see Conjecture 1), which is a real number in all cases, is contained in $I_{f, D}$ if the conjecture holds. The intersection $\bigcap_{D} I_{f, D}$ is nonempty for weight- $k$ cuspidal Hecke eigenforms that are not contained in the Maass Spezialschar when $20 \leq k<40$.

In Section 4, we present two further applications. First, we state a conjecture on the maximal discriminant of a pivot set for the Fourier expansion of a basis. This has already been considered in a recent preprint [13]. We are able to provide evidence of better asymptotic behavior. Second, we consider the rational Hecke action on the spaces of weight $k$ forms as mentioned above.

Although the construction of the generating set was motivated by computational needs, this set turns out to have interesting arithmetic properties. We will present these at the end of Section 4.

Throughout this paper, we consider only spaces of rational modular forms. In particular, we are not concerned with integral modular forms. A basis for the module of integral Siegel modular forms can easily be deduced from our results, and might be of some use.

\section{Generating spaces by products of elements in the Maass Spezialschar}

The well-known Igusa generators for the ring of classical Siegel modular forms associated to the full modular group of degree two (see [9]) are elements of the Maass Spezialschar. Multiplication of multidimensional Fourier expansions is computationally very expensive. Thus, an important question arises: how many multiplications will we need to calculate a basis element of a given space of modular forms of weight $k$ ? Using Igusa generators, the answer is clearly $O(\log (k))$. For example, to calculate the Fourier expansion of a generic weight 30 form, we have to perform more than 15 multiplications and the longest product involves four multiplications. 
Since it is rather cheap to calculate Fourier expansions of elements in the Maass Spezialschar, we do not need to restrict to the aforementioned generators of the ring, but we can consider all elements of this space. Indeed, up to weight 18, the Maass Spezialschar is equal to the spaces of modular forms. For every higher weight, it is a proper subspace. Surprisingly, using this enlarged set of generators, we are able to restrict to products with at most two factors. Using such a basis to calculate the Fourier expansion of a generic weight 30 form, we need six multiplications and the longest product involves two factors.

From now on we assume that Siegel modular forms are classical unless otherwise stated. To state a precise conjecture, denote the Jacobian modular group of degree one by $\Gamma_{J}$ and the full Siegel modular group of degree $g$ by $\Gamma_{g}$. For a detailed discussion of Jacobi forms, see [7]. Let $\left[\Gamma_{g}, \operatorname{det}^{k}\right]$ and $\left[\Gamma_{g}, \operatorname{det}^{k}\right]_{0}$ be the spaces of modular forms and cusp forms of weight $k$, respectively. Analogously, denote the space of Jacobi forms of index $m$ and weight $k$ by $\left[\Gamma_{J}, m, k\right]$. Let $V f$ denote Gritsenko's arithmetic lift of a Jacobi form $f$ (see [8]).

Conjecture 2. We have

$$
\bigoplus_{k \geq 0,2 \mid k}\left[\Gamma_{2}, \operatorname{det}^{k}\right]=\left(\bigoplus_{k \geq 0,2 \mid k} V\left[\Gamma_{J}, 1, k\right]\right)^{2} .
$$

REMARK 3. The reader may wonder if there is an obvious obstruction to our conjecture that arises from dimensional considerations. The spaces of formal products of Maass lifts have dimension asymptotically growing as $k^{5}$, while the spaces of Siegel modular forms have dimension asymptotically growing as $k^{4}$.

REMARK 4. This conjecture is an analog of the fact that the spaces for weight $k$ are spanned by $E_{k}$ and $E_{i} E_{k-i}$ for $i \in\{4, \ldots, k-4\}$ for the full elliptic modular group. In this case, even the relations can be deduced with help of the theory of periods (see [11]).

REMARK 5. The paramodular analog of Conjecture 2 has to include symmetrizations of paramodular forms of lower level. This is already relevant for paramodular forms of level two, where symmetrizations of Siegel modular forms for the full modular group suffice to span the space together with Gritsenko lifts for low weights. Notice that there are paramodular forms of weight two for sufficiently high levels, as was shown by Poor and Yuen in [13]. Gritsenko suggested that Gritsenko lifts with a character, that also exist for weight 1 , might fill this gap.

By explicit computation, we can prove the following theorem.

TheOrem 6. Conjecture 2 holds up to weight 172.

In Table 1, we present some of the generators that we calculated. To explain the table, we fix some notation. The Maass Spezialschar is the image of

$$
\left[\Gamma_{J}, 1, k\right] \cong\left[\Gamma_{1}, \operatorname{det}^{k}\right] \times\left[\Gamma_{1}, \operatorname{det}^{k+2}\right]_{0}
$$


TABLE 1. Products generating the spaces of Siegel modular forms, ignoring the Maass Spezialschar.

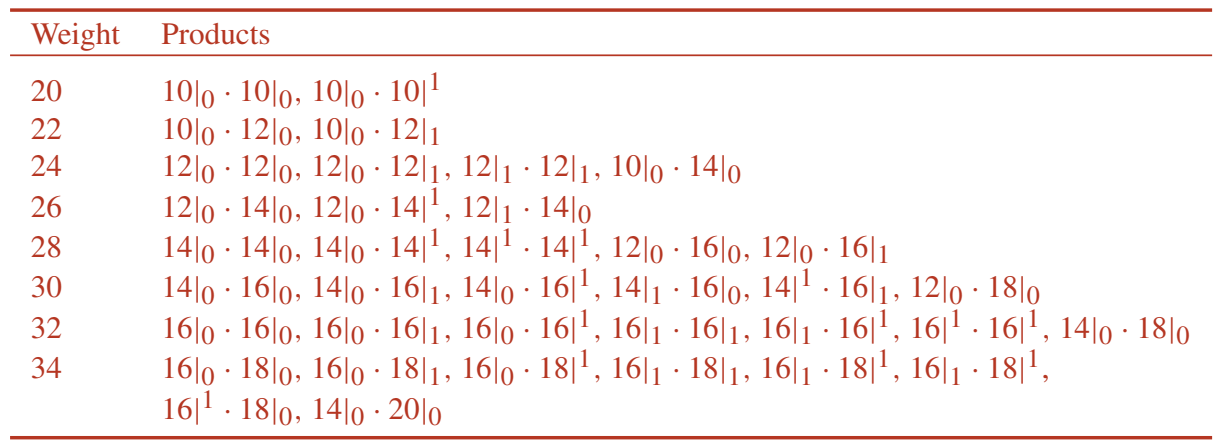

Every space $\left[\Gamma_{1}, \operatorname{det}^{k}\right]$ admits a Victor-Miller basis, which is canonically enumerated. We denote this basis by $\left\{f_{k, i}: i \in\left\{0, \ldots, \operatorname{dim}\left[\Gamma_{1}, \operatorname{det}^{k}\right]-1\right\}\right\}$. The lift of $\left(f_{k, i}, 0\right)$ is denoted by $\left.k\right|_{i}$ and the lift of $\left(0, f_{k+2, i}\right)$ is denoted by $\left.k\right|^{i}$. In the table, we have listed only elements which do not belong to the Maass Spezialschar. Together with $V\left[\Gamma_{J}, 1, k\right]$ they span $\left[\Gamma_{2}, \operatorname{det}^{k}\right]$.

\section{The Böcherer conjecture}

To verify the Böcherer conjecture for nonrational eigenforms, a suitable generating set for the spaces of Siegel modular forms was needed. This motivated the investigations in Section 2.

In this section, we approximate the central values of the twisted spinor $L$-function with a bound on the approximation error. This yields very small intervals for the constant $c_{f}$. Since they have nonempty intersection, the calculations in this section support Conjecture 1.

Consider a cuspidal eigenform $f \in\left[\Gamma_{2}\right.$, det $\left.{ }^{k}\right]$ and a primitive Dirichlet character $\chi_{D}=\left(\frac{D}{.}\right)$. We revise the approximation of the twisted spinor $L$-function $Z_{f, D}$ at $s \in \mathbb{C}$ based on a series representation found by Kohnen (see [10]). We denote the $n$th coefficient of the spinor $\zeta$-function by $\lambda_{f}(n)$. Then

$$
Z_{f, D}(s)=\sum_{n=1}^{\infty} g_{D}(n) \lambda_{f}(n) \chi_{D}(n),
$$

where

$$
\begin{aligned}
g_{D}(n)=2(2 \pi /|D|)^{2-k+2 s} n^{-k / 2} /(\Gamma(s) \Gamma(s-k+2)) & \\
& \times \int_{n}^{\infty} K_{k-2}(4 \pi \sqrt{y} /|D|)\left((y / n)^{3 k / 2-2-s}+(y / n)^{s-k / 2}\right) d y .
\end{aligned}
$$

By $K_{k-2}$ we mean the $K$-Bessel function with parameter $k-2$. 
This series can be approximated by suitably truncating it:

$$
\widetilde{Z}_{f, D}(s, P, N)=\sum_{n \in S(N, P)} g_{D}(n) \lambda_{f}(n) \chi_{D}(n),
$$

where $S(N, P)$ is the set of all positive integers greater than $N$ all of whose prime divisors are less than $P$. When $s=k-1$, a bound for the approximation error has already been given by Kohnen and Kuß:

$$
\begin{aligned}
& \left|Z_{f, D}(k-1)-\widetilde{Z}_{f, D}(k-1, P, N)\right| \\
& \quad \leq \eta_{f}(N, P, D, s) \\
& \quad:=\sum_{\substack{N>p>P \\
1 \leq \nu \leq N / p}} 4 p^{k-3 / 2}\left|\lambda_{f}(v)\right| \widetilde{g}_{D}(v p) \\
& \quad+4(2 \pi /|D|)^{k} / \Gamma(k-1) \int_{N+1}^{\infty} K_{k-2}(4 \pi \sqrt{y} /|D|) y^{(k+1) / 2}(y-N) d y .
\end{aligned}
$$

The function $\widetilde{g}_{D}$ is defined as follows:

$$
\widetilde{g}_{D}(n):=4(2 \pi /|D|)^{k} / \Gamma(k-1) \int_{n}^{\infty} K_{k-2}(4 \pi \sqrt{y} /|D|)(y / n)^{k / 2-1} .
$$

Note that this bound is far from optimal. So we also use a second estimate,

$$
\tilde{\eta}_{f}\left(P, N, P^{\prime}, N^{\prime}, D, s\right)=\left|\tilde{Z}_{f, D}(s, P, N)-\tilde{Z}_{f, D}\left(s, P^{\prime}, N^{\prime}\right)\right|,
$$

with suitable $P^{\prime}$ and $N^{\prime}$. Later we will have to introduce a correction factor $\kappa$, since $\tilde{\eta}$ might become too small if the series converges slowly. Nevertheless, the bounds $\kappa \tilde{\eta}_{f}$ obtained in this way are much tighter in general.

In the course of these calculations, we will take $P=1500, N=7999, P^{\prime}=1000$, and $N^{\prime}=3999$, and neglect these parameters in notation. We will also omit the parameter $s$, if $s=k-1$.

Denote the Fourier expansion of $f \in\left[\Gamma_{2}, \operatorname{det}^{k}\right]$ by

$$
f(z)=\sum_{t} a_{f}(t) \exp (2 \pi i \cdot \operatorname{tr}(t z)) .
$$

Here tr is the usual trace of a matrix. We will use Andrianov's formula for the Hecke eigenvalues (see [10] for an easy-to-read statement). To do so, we need to assume that

$$
a_{f}\left(\begin{array}{cc}
1 & 1 / 2 \\
1 / 2 & 1
\end{array}\right) \neq 0 \text {. }
$$

This assumption holds for all the forms that we consider.

We have to calculate the Fourier expansion of forms $f$ up to discriminants $-\sqrt{8000}$. This is no problem at all. But we also have to calculate certain Fourier coefficients of discriminant up to $-3 \times 1499^{2} \approx-6.75 \times 10^{6}$. Namely, we have to calculate the 
Fourier coefficients associated to

$$
\left(\begin{array}{cc}
1 & p / 2 \\
p / 2 & p^{2}
\end{array}\right)
$$

This is actually a very hard problem.

We briefly describe the technical details of our method. By the results of the preceding section, we can use a basis which consists of products of at most two elements of the Maass Spezialschar in all cases considered. We have to compute $\eta^{-6}$. Notice that this is the most expensive part of the computation of the Fourier expansion of the lifts and we precalculate it. Multiplication of such big polynomials with only positive coefficients using Fourier transforms consumes too much memory. Here, we combine a multimodular approach with ordinary $\mathcal{O}\left(n^{2}\right)$ multiplication, which admits easy hard-drive caching.

Assume that we have calculated the elements of the Maass Spezialschar. Let us now focus on a nontrivial product $g=g_{1} \cdot g_{2}$. We have to calculate certain Fourier coefficients $a_{g}(t)=\sum_{t_{1}+t_{2}=t} a_{g_{1}}\left(t_{1}\right) \cdot a_{g_{2}}\left(t_{2}\right)$. Since $a_{g_{i}}\left(t_{i}\right)$ depends only on $d_{i}:=\operatorname{det}\left(t_{i}\right)$ and $\epsilon_{i}:=\operatorname{gcd}\left(t_{i}\right)$ (here $\left.i=1,2\right)$, we may write

$$
a_{g}(t)=\sum_{\epsilon_{1}, \epsilon_{2}, d_{1}, d_{2}} v\left(\epsilon_{1}, \epsilon_{2}, d_{1}, d_{2}\right) a_{g_{1}}\left(\epsilon_{1}, d_{1}\right) a_{g_{2}}\left(\epsilon_{2}, d_{2}\right) .
$$

The function $v: \mathbb{Z}^{4} \rightarrow \mathbb{N}$ can be precalculated. This formula saves a lot of time, as the same numbers do not need to be multiplied many times. See the code, which is available on the author's homepage, for more details.

We calculate the central values of twisted spinor $L$-series associated to cuspidal Hecke eigenforms which do not belong to the Maass Spezialschar of even weight $k$ up to 40, and for all discriminants $D$ strictly between 0 and -300 . The relevant quotients are denoted by $c_{f}^{\prime}(D):=\tilde{Z}_{f, D}(s, P, N) / B_{f}(D)$. The factor $B_{f}(D)$ is essentially the right-hand side of the Böcherer conjecture, as defined in the introduction. The results support the Böcherer conjecture. However, for large discriminants, they are not very precise. A brief summary is given in Tables 3 and 4. For the complete data, download the relevant files on the author's homepage. Here is a precise statement.

THEOREM 7. Let $f$ be a weight-k cuspidal Hecke eigenform that is not an element of the Maass Spezialschar, where $20 \leq k<40$. Then the intervals

$$
\left[c_{f}^{\prime}(D)-\eta_{f}(D) / B_{f}(D), c_{f}^{\prime}(D)+\eta_{f}(D) / B_{f}(D)\right]
$$

for discriminants $D$ strictly between 0 and -300 , have nonempty intersection. If $\kappa_{f}$ is chosen according to Table 2, then the intervals

$$
\left[c_{f}^{\prime}(D)-\kappa_{f} \tilde{\eta}_{f}(D) / B_{f}(D), c_{f}^{\prime}(D)+\kappa_{f} \tilde{\eta}_{f}(D) / B_{f}(D)\right]
$$

also have nonempty intersection. 
TABLE 2. Correction factors $\kappa$ for weight- $k$ forms in Theorem 7.

\begin{tabular}{lllllllllllll}
\hline$k$ & 20 & 22 & $24 a$ & $24 b$ & $26 a$ & $26 b$ & 28 & 30 & 32 & 34 & 36 & 38 \\
\hline$\kappa_{f}$ & 180 & 90 & 70 & 45 & 400 & 220 & 90 & 150 & 120 & 2300 & 85 & 400 \\
\hline
\end{tabular}

TABLE 3. Numerical estimates $c_{f}^{\prime}(D)$ of the constant $c_{f}$ occurring in the Böcherer conjecture.

\begin{tabular}{|c|c|c|c|c|}
\hline Weight & $D$ & $c_{f}^{\prime}(D)$ & $\eta(D) / B_{f}(D)$ & $\tilde{\eta}(D) / B_{f}(D)$ \\
\hline \multirow[t]{4}{*}{20} & -3 & $2.067215202868765 \times 10^{11}$ & $8.7123 \times 10^{-41}$ & $2.4462 \times 10^{-31}$ \\
\hline & -4 & $2.067215202868765 \times 10^{11}$ & $2.6633 \times 10^{-24}$ & $2.9904 \times 10^{-17}$ \\
\hline & -15 & $2.067220228408808 \times 10^{11}$ & $8.2915 \times 10^{25}$ & $1.8868 \times 10^{6}$ \\
\hline & -20 & $2.066999728524787 \times 10^{11}$ & $3.3599 \times 10^{33}$ & $2.2640 \times 10^{8}$ \\
\hline \multirow[t]{4}{*}{28} & -3 & $5.105310601780946 \times 10^{14}$ & $5.6429 \times 10^{-32}$ & $3.3222 \times 10^{-22}$ \\
\hline & -4 & $5.105310601780946 \times 10^{14}$ & $9.7141 \times 10^{-17}$ & $3.4976 \times 10^{-10}$ \\
\hline & -15 & $5.105287106899537 \times 10^{14}$ & $2.0716 \times 10^{45}$ & $1.2487 \times 10^{11}$ \\
\hline & -20 & $5.108464527256237 \times 10^{14}$ & $8.7051 \times 10^{53}$ & $2.0228 \times 10^{12}$ \\
\hline \multirow[t]{4}{*}{30} & -3 & $7.494659605198417 \times 10^{15}$ & $6.3380 \times 10^{-30}$ & $3.3652 \times 10^{-21}$ \\
\hline & -4 & $7.494659605198417 \times 10^{15}$ & $1.4559 \times 10^{-13}$ & $3.3121 \times 10^{-7}$ \\
\hline & -15 & $7.493428754840905 \times 10^{15}$ & $4.6698 \times 10^{50}$ & $3.8657 \times 10^{12}$ \\
\hline & -20 & $7.525312838702049 \times 10^{15}$ & $2.9997 \times 10^{60}$ & $2.7895 \times 10^{14}$ \\
\hline \multirow[t]{4}{*}{32} & -3 & $1.205163535250015 \times 10^{17}$ & $6.3368 \times 10^{-28}$ & $3.5683 \times 10^{-18}$ \\
\hline & -4 & $1.205163535250015 \times 10^{17}$ & $1.5997 \times 10^{-12}$ & $6.5727 \times 10^{-7}$ \\
\hline & -15 & $1.205732652809925 \times 10^{17}$ & $3.2290 \times 10^{57}$ & $3.7921 \times 10^{15}$ \\
\hline & -20 & $1.205060112394051 \times 10^{17}$ & $2.9225 \times 10^{64}$ & $7.7758 \times 10^{13}$ \\
\hline \multirow[t]{4}{*}{34} & -3 & $1.086138503038145 \times 10^{18}$ & $5.6918 \times 10^{-26}$ & $2.5842 \times 10^{-17}$ \\
\hline & -4 & $1.086138503038145 \times 10^{18}$ & $9.1579 \times 10^{-10}$ & $6.7761 \times 10^{-4}$ \\
\hline & -15 & $1.086049155614554 \times 10^{18}$ & $1.3262 \times 10^{61}$ & $9.5845 \times 10^{15}$ \\
\hline & -20 & $1.084637644518613 \times 10^{18}$ & $9.1134 \times 10^{70}$ & $3.1879 \times 10^{16}$ \\
\hline \multirow[t]{4}{*}{36} & -3 & $1.684497849534415 \times 10^{19}$ & $4.6300 \times 10^{-24}$ & $5.5959 \times 10^{-16}$ \\
\hline & -4 & $1.684497849534415 \times 10^{19}$ & $6.5481 \times 10^{-9}$ & $4.5361 \times 10^{-3}$ \\
\hline & -15 & $1.704113931864776 \times 10^{19}$ & $4.0039 \times 10^{68}$ & $1.2502 \times 10^{18}$ \\
\hline & -20 & $1.683266203530922 \times 10^{19}$ & $3.3778 \times 10^{75}$ & $5.0592 \times 10^{16}$ \\
\hline \multirow[t]{4}{*}{38} & -3 & $6.357076618815059 \times 10^{19}$ & $3.4351 \times 10^{-22}$ & $1.1738 \times 10^{-14}$ \\
\hline & -4 & $6.357076618815059 \times 10^{19}$ & $1.8281 \times 10^{-7}$ & $4.3949 \times 10^{-1}$ \\
\hline & -15 & $6.517241676742821 \times 10^{19}$ & $8.0534 \times 10^{73}$ & $2.9605 \times 10^{18}$ \\
\hline & -20 & $6.375510867491227 \times 10^{19}$ & $1.6586 \times 10^{81}$ & $7.7408 \times 10^{17}$ \\
\hline
\end{tabular}


TABLE 4. Some values of $\left|\log \left(c_{f}^{\prime}(D) / c_{f}^{\prime}(-3)\right)\right|$.

\begin{tabular}{llllllll}
\hline$D \backslash$ weight & 20 & 28 & 30 & 32 & 34 & 36 & 38 \\
\hline-4 & 0.0000 & 0.0000 & 0.0000 & 0.0000 & 0.0000 & 0.0000 & 0.0000 \\
-7 & 0.0000 & 0.0000 & 0.0000 & 0.0000 & 0.0000 & 0.0000 & 0.0000 \\
-20 & 0.0001 & 0.0006 & 0.0041 & 0.0001 & 0.0014 & 0.0007 & 0.0029 \\
-103 & 1.3565 & 0.1592 & 3.3038 & 0.4526 & 1.2287 & 1.0546 & 1.9854 \\
-203 & 0.2641 & 0.4993 & 2.2404 & 0.1465 & 0.6835 & 0.6584 & 0.4231 \\
-299 & 0.2152 & 0.2511 & 0.1197 & 0.7238 & 1.1443 & 0.2187 & 0.2517 \\
\hline
\end{tabular}

REMARK 8. We note that the only critical value of $L$-series attached to Siegel modular forms is $k-1$. Hence Deligne's conjecture [6] predicts that the central value is, up to an algebraic number, the determinant of the period matrix attached to the eigenform $f$. This is captured in the constant $c_{f}$, but we know of no way to extract this determinant from $c_{f}$.

\section{Two further applications}

As mentioned in the introduction, one motivation for finding a basis as described in Section 2 was to provide computational access to high weights. Indeed, on an ordinary Sun server we are able to compute the Fourier expansions of the basis sufficiently precisely to apply Hecke operators in a few seconds. In this section, we present two applications which become amenable using the new bases.

4.1. The minimal discriminant of pivot sets. One often encounters modular forms in other fields of mathematics. In order to use modular forms as a tool of investigation in these fields, it is crucial to express them in terms of a given basis. This is often done by comparing Fourier coefficients. Sometimes it is computationally very expensive to calculate the Fourier expansions. Since the costs of this computation grow rapidly in terms of the discriminant of the Fourier index, it is natural to ask the following question: up to which discriminant does one have to calculate the Fourier expansion of a Siegel modular form of degree two to uniquely determine it?

More precisely, we fix $k$ and denote the Fourier expansion of a modular form $f$ by $\sum a_{f}(t) \exp (2 \pi i \cdot \operatorname{tr}(t z))$. Now, we consider $D(\mathfrak{p})=\min _{i}\left(-4 \operatorname{det}\left(t_{i}\right)\right)$ the minimal discriminant associated to any set of pivot indices $\mathfrak{p}=\left\{t_{i}\right\}_{i}$ of $\left[\Gamma_{2}, \operatorname{det}^{k}\right]$. By pivot indices, we mean a set of indices $\mathfrak{p}=\left\{t_{i}\right\}_{i}$ such that the rank of the matrix $\left(a_{f}\left(t_{i}\right)\right)_{i, f}$, where $f$ runs over a basis of $\left[\Gamma_{2}, \operatorname{det}^{k}\right]$, is equal to $\operatorname{dim}\left[\Gamma_{2}, \operatorname{det}^{k}\right]$. Now the question can be formulated as follows. Can we express $\max _{\mathfrak{p}} D(\mathfrak{p})$ in terms of $k$ ?

Using the basis presented in the preceding section, we can give a partial answer to this question. The double logarithmic plot in Figure 1 of the data from Table 5 reveals that for high weights $k$ the slope tends to 2. This supports the following conjecture. 


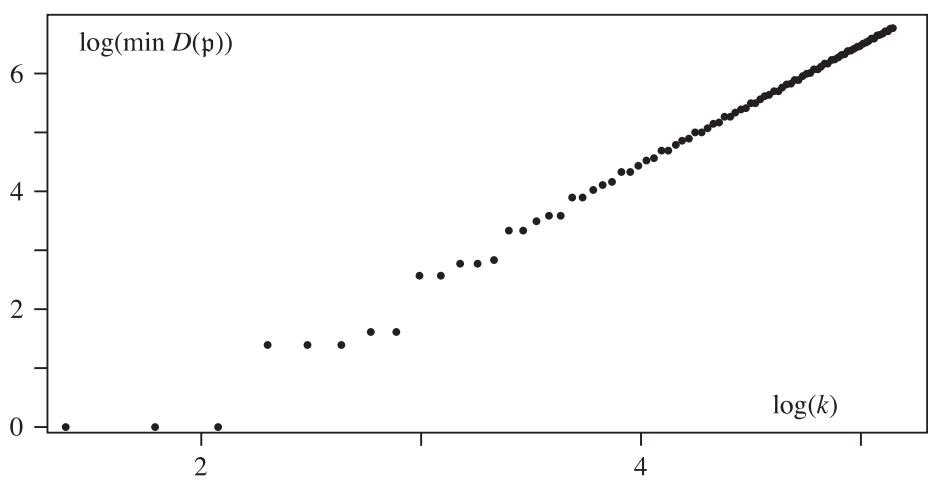

FIGURE 1. Double logarithmic plot of maximal minimal discriminants for weights up to 172.

TABLE 5. Maximal minimal discriminants of pivot sets for weights $100 \leq k<160$.

\begin{tabular}{llllll}
\hline Weight & $\max _{\mathfrak{p}} D(\mathfrak{p})$ & Weight & $\max _{\mathfrak{p}} D(\mathfrak{p})$ & Weight & $\max _{\mathfrak{p}} D(\mathfrak{p})$ \\
\hline 100 & 301 & 120 & 433 & 140 & 589 \\
102 & 301 & 122 & 436 & 142 & 596 \\
104 & 320 & 124 & 456 & 144 & 616 \\
106 & 337 & 126 & 477 & 146 & 641 \\
108 & 341 & 128 & 481 & 148 & 645 \\
110 & 364 & 130 & 508 & 150 & 676 \\
112 & 365 & 132 & 513 & 152 & 685 \\
114 & 385 & 134 & 533 & 154 & 705 \\
116 & 404 & 136 & 556 & 156 & 732 \\
118 & 408 & 138 & 560 & 158 & 736 \\
\hline
\end{tabular}

CONJECTURE 9. Fix $k$ and let $\mathfrak{p}$ run over all sets of pivot indices associated to weight$k$ forms. Then

$$
\max _{\mathfrak{p}} D(\mathfrak{p})=-c k^{2}+\mathcal{O}(1) \quad \text { as } k \rightarrow \infty,
$$

where $c \approx 184 / 79^{2}<0.03$.

REMARK 10. Note that the analogous result for $\left[\Gamma_{1}, \operatorname{det}^{k}\right]$ is $\max _{\mathfrak{p}} D(\mathfrak{p})=-k$, due to the Victor-Miller construction. In a recent preprint, Poor and Yuen show a similar result that implies that $c \leq 8 / 225 \approx 0.035$ (see [13]).

4.2. Irreducibility of the Hecke action. Let $K:\left[\Gamma_{1}, k\right] \rightarrow\left[\Gamma_{2}, k\right]$ be the KlingenEisenstein lift. The Hecke invariant splitting of the spaces of Siegel modular forms

$$
\left[\Gamma_{2}, k\right]=K\left[\Gamma_{1}, k\right]_{0} \oplus V\left[\Gamma_{J}, 1, k\right] \oplus S_{k}^{\prime}
$$

is well known. 
In analogy to the degree-one case, one might expect that $S_{k}^{\prime}$ is irreducible with respect to the rational Hecke action for all $k$. But computations by Skoruppa in [17] revealed that $S_{k}^{\prime}$ is reducible if $k \in\{24,26\}$. He found the aforementioned eigenforms $\Upsilon_{k a}, \Upsilon_{k b}$. We mention that the normalization of $\Upsilon_{k *}$ was chosen such that all Fourier coefficients were integral and coprime.

Nevertheless, the conjecture that the Hecke action on spaces of Siegel modular forms should be 'as irreducible as possible' is commonly supposed to hold true. Using the computational advantages of the basis presented in Section 2, we were able to prove the following result.

THEOREM 11. The spaces $S_{k}^{\prime}$ are irreducible with respect to the Hecke action for all even $k$ such that $28 \leq k \leq 150$.

REMARK 12. No attempt was made to consider the odd-weight case, although in principle the computations are feasible using Wronskians. See [15] for a detailed treatment.

To prove this, the author calculated the matrix associated to the Hecke operator $T$ (2) with respect to the basis above using Sage, and then calculated its minimal polynomial using Magma.

4.3. Interesting properties of the basis. The bases presented in Table 1 have surprising properties. They are canonical in the sense that the maximum of the weights of all lifts involved in nontrivial products is minimal within the set of all possible bases of that kind.

We can read off the noteworthy fact that $\left.\left.14\right|_{1} \cdot 16\right|_{1}$ is contained in the sixdimensional space spanned by the Maass Spezialschar together with the three elements

$$
\left.\left.14\right|_{0} \cdot 16\right|_{0},\left.\left.\quad 14\right|_{0} \cdot 16\right|_{1},\left.\left.\quad 14\right|_{0} \cdot 16\right|^{1},
$$

whereas $\operatorname{dim}\left[\Gamma_{2}, \operatorname{det}^{30}\right]=11$. Hence the products we use to span the spaces of Siegel modular forms satisfy more linear relations than one might expect in general.

Next, we consider the two Hecke eigenforms $\Upsilon_{24 a}$ and $\Upsilon_{24 b}$ of weight 24 normalized as in [17]. Expressing them in terms of the given basis yields two vectors $v_{a}, v_{b} \in \mathbb{Q}^{8}$. It is surprising that $\left(v_{a}\right)_{i}=0$ exactly when $\left(v_{b}\right)_{i}=0$, as one can see in Table 6. An analogous result holds for the Hecke eigenforms $\Upsilon_{26 a}$ and $\Upsilon_{26 b}$ (see Table 7). This suggests that the bases that we constructed are indeed connected to periods, as mentioned in Remark 4.

There is one further phenomenon which presumably is of arithmetic interest and might reflect the above connection. The denominators of the entries in these vectors tend to be smooth. Recall that $\Upsilon_{24 *}$ and $\Upsilon_{26 *}$ are canonically normalized in the sense that they are primitive integral eigenforms.

REMARK 13. We remark that the framework used to calculate this basis as well as the applications are available on the author's homepage. This framework is derived from a set of tools that is presented in [15]. 
TABLE 6. Coordinates of the exceptional eigenforms of weight 24 with respect to the basis in Table 1.

\begin{tabular}{ll}
\hline$\Upsilon_{24 a}$ & $\Upsilon_{24 b}$ \\
\hline 0 & 0 \\
$-29^{2} \cdot 2237 / 2^{5} \cdot 3^{4} \cdot 5 \cdot 7 \cdot 13$ & $416761 / 2^{3} \cdot 3^{5} \cdot 5^{2} \cdot 7$ \\
$-52956193 / 2^{2} \cdot 3^{3} \cdot 5 \cdot 7 \cdot 13$ & $-11 \cdot 83 \cdot 4987 / 3^{4} \cdot 5^{2} \cdot 7$ \\
$-227 \cdot 2969 / 2^{5} \cdot 3^{4} \cdot 5 \cdot 7 \cdot 13$ & $937 \cdot 947 / 2^{3} \cdot 3^{5} \cdot 5^{2} \cdot 7$ \\
0 & 0 \\
$11 \cdot 157 / 2^{6} \cdot 3^{5} \cdot 5 \cdot 7 \cdot 13$ & $13 \cdot 83 / 2^{4} \cdot 3^{6} \cdot 5^{2} \cdot 7$ \\
$2^{2} \cdot 5 \cdot 11 \cdot 157 \cdot 661 / 3$ & $-2^{4} \cdot 5 \cdot 7 \cdot 13^{3} \cdot 83 / 3^{2}$ \\
0 & 0 \\
\hline
\end{tabular}

TABLE 7. Coordinates of the exceptional eigenforms of weight 26 with respect to the basis in Table 1.

\begin{tabular}{ll}
\hline $71 \cdot 139 \cdot \Upsilon_{26 a}$ & $71 \cdot 139 \cdot \Upsilon_{26 b}$ \\
\hline 0 & 0 \\
$10718579 / 2^{3} \cdot 13^{2}$ & $-3 \cdot 5 \cdot 54059 / 2^{3}$ \\
$61 \cdot 79 \cdot 3967087 / 2^{4} \cdot 3 \cdot 5^{2} \cdot 7 \cdot 13^{2}$ & $-2251 \cdot 9923 / 2^{4} \cdot 3 \cdot 5$ \\
$3 \cdot 1703358596089 / 2 \cdot 5^{2} \cdot 7 \cdot 13^{2}$ & $-3 \cdot 191 \cdot 10784339 / 2 \cdot 5$ \\
0 & 0 \\
$-11 \cdot 29 \cdot 839 / 2 \cdot 5^{2} \cdot 7 \cdot 13^{2}$ & $4177 / 2^{3} \cdot 3 \cdot 5$ \\
$11 \cdot 29 \cdot 37 \cdot 167 / 2^{5} \cdot 3 \cdot 7 \cdot 13$ & $-5 \cdot 7 \cdot 13 \cdot 19 \cdot 37 / 2^{5} \cdot 3$ \\
\hline
\end{tabular}

\section{Acknowledgements}

Major parts of this work were done at the Lehrstuhl A für Mathematik, RWTH Aachen University, 52056 Aachen, Germany. The author wishes to express his thanks to the Lehrstuhl B für Mathematik, RWTH Aachen University, for the computational resources that they provided.

\section{References}

[1] J. Bergström, C. Faber and G. van der Geer, 'Siegel modular forms of genus 2 and level 2: cohomological computations and conjectures', Preprint, 2008.

[2] S. Böcherer, 'Bemerkungen über die Dirichletreihen von Koecher und Maaß (remarks on the Dirichlet series of Koecher and Maaß)', Math. Gottingensis 68 (1986), 1-36.

[3] S. Böcherer and R. Schulze-Pillot, 'The Dirichlet series of Koecher and Maaß and modular forms of weight 3/2', Math. Z. 209 (1992), 273-287.

[4] W. Bosma, J. Cannon and C. Playoust, 'The Magma algebra system. I. The user language', J. Symbolic Comput. 24 (1997), 235-265.

[5] A. Brumer and K. Kramer, 'Abelian surfaces over $\mathbb{Q}$ ', Preprint, 2009.

[6] P. Deligne, 'Valeurs de fonctions $L$ et périodes d'intégrales', in: Automorphic Forms, Representations and L-functions (Proc. Sympos. Pure Math., Oregon State Univ., Corvallis, Ore., 1977), Part 2, Proceedings of Symposia in Pure Mathematics, XXXIII (American Mathematical Society, Providence, RI, 1979), pp. 313-346. 
[7] M. Eichler and D. Zagier, The Theory of Jacobi Forms (Birkhäuser, Boston, MA, 1985).

[8] V. A. Gritsenko, 'Modulformen zur Paramodulgruppe und Modulräume der abelschen Varietäten', Math. Gottingensis 12 (1995), 1-89.

[9] J. Igusa, 'On Siegel modular forms of genus two', Amer. J. Math. 84 (1962), 175-200.

[10] W. Kohnen and M. Kuß, 'Some numerical computations concerning spinor zeta functions in genus 2 at the central point', Math. Comp. 71(240) (2002), 1597-1607.

[11] W. Kohnen and D. Zagier, Modular forms with rational periods', Modular forms, Durham Symposium, England, 1983, 197-249, 1984.

[12] A. Krieg and M. Raum, 'The functional equation for the twisted spinor- $L$-function of genus 2', arXiv:0907.2767 [math.NT].

[13] C. Poor and D. Yuen, 'Paramodular cups forms', 2009, arXiv:0912.0049v1 [math.NT].

[14] M. Raum, 'A computational approach to the Böcherer conjecture', talk at the AKLS meeting Cologne, May 5th 2010.

[15] M. Raum, N. Ryan, N. P. Skoruppa and G. Tornaría, 'Theoretical and algorithmic aspects of an implementation of siegel modular forms', Preprint, 2010.

[16] N. C. Ryan and G. Tornaría, 'A Böcherer-type conjecture for paramodular forms', 2010, http://arxiv.org/abs/1006.1582v1 [math.NT].

[17] N. P. Skoruppa, 'Computations of Siegel modular forms of genus two', Math. Comp. 58(197) (1992), 381-398.

[18] W. A. Stein et al., 'Sage mathematics software (version 4.1.2)', 2009, http://www.sagemath.org.

MARTIN RAUM, MPI für Mathematik, Vivatsgasse 7, 53111 Bonn, Germany e-mail: MRaum@mpim-bonn.mpg.de 